\title{
Exercício físico e sua influência na saúde sexual
}

\author{
Physical exercise influence on sexual health
}

Gabriela Maria Dutra de Carvalho, ${ }^{1}$ Ana Inês Gonzáles, ${ }^{1}$ Sabrina Weiss Sties, ${ }^{1}$ Daiane Pereira Lima,

Almir Schmitt Neto, Tales de Carvalho. ${ }^{1}$

Universidade do Estado de Santa Catarina (UDESC), Florianópolis, SC, Brasil.

Recebido em: abril 2015 / Aceito em: abril 2015

anainesgonzales@gmail.com

\section{RESUMO}

Assim como a saúde em geral, o desempenho sexual depende do bem estar físico, mental e social, sendo influenciado pelo estilo de vida, contexto no qual merece destaque a atividade física. Neste ensaio apresentamos alguns dos mecanismos pelos quais a prática regular de exercícios físicos por múltiplos mecanismos fisiológicos contribui para a saúde do corpo e da mente, e defendemos a hipótese de que a dança de salão pode ser considerada como excelente opção quando se objetiva ao mesmo tempo o aprimoramento da função cardiovascular e sexual.

Palavras-chave: Qualidade de Vida; Disfunção Erétil; Sexualidade.

\section{ABSTRACT}

Just like health in general, sexual performance depends on physical, mental and social wellbeing, and it is influenced by lifestyle, so in this context physical activity has to be highlighted. In this study we present some of the mechanisms which regular physical exercise by multiple physiological mechanisms improves body and mind health. Furthermore we hereby defend the hypothesis that ballroom dancing can be considered an excellent option when aiming the simultaneous improvement of cardiovascular and sexual function.

Keywords: Quality of Life; Erectile Dysfunction, Sexuality.

\section{INTRODUÇÃO}

A sexualidade se expressa em valores e comportamentos, como o desejo, o prazer e a corporeidade, que são frequentemente relacionadas à qualidade de vida. Considerando que, segundo a Organização Mundial de Saúde (OMS) ter boa saúde significa estar bem do ponto de vista físico, mental e social, os termos saúde e qualidade de vida devem ser considerados termos coadjuvantes. A boa expressão da sexualidade depende da saúde vista de uma forma ampla, podendo-se afirmar que saúde, qualidade de vida e função sexual estão intimamente relacionadas. Nesse contexto, a expressão da sexualidade, que contribui decisivamente para a qualidade e a longevidade das relações afetivas, deve ser considerada um componente relevante da saúde global $^{1-3}$.

A disfunção sexual (DS) é um problema de saúde de grande relevância, que afeta igualmente homens e mulheres, sendo definida pela Organização Mundial da Saúde como a situação em que um indivíduo é incapaz de participar de uma relação sexual como gostaria ${ }^{4}$ No sexo masculino, a discussão se restringe, frequentemente, à disfunção erétil (DE) caracteriza-se pela inabilidade persistente ou recorrente para atingir ou manter a ereção suficiente para completar a relação sexual ${ }^{5}$. Existem diversas definições para a disfunção sexual feminina (DSF), condição frequentemente descrita como sendo a "diminuição persistente ou recorrente do desejo sexual, da excitação sexual, da dificuldade ou inabilidade para atingir o orgasmo, e da ocorrência de dor, durante a relação sexual", sendo esta última situação denominada dispaurenia ${ }^{6}$ e a sua investigação bem complexa ${ }^{7}$.

Os fatores que causam as doenças crônicas não transmissíveis (doenças cardiovasculares, câncer, diabetes, etc.) são os mesmos que podem causar a DS, que devem ser considerados importantes preditores de eventos cardiovasculares, principalmente, quando ocorre em indivíduos mais jovens, com menos de 60 anos. A DE deve ser considerada um relevante fator relacionado à doença cardiovascular, assim como o diabetes, a dislipidemia, o sedentarismo e o tabagismo, reconhecidos como importantes fatores de risco para doença cardíaca. Essa doença costuma preceder em 2 a 
3 anos a angina e em 3 a 5 anos os eventos cardiovasculares maiores, como o infarto do miocárdio e a morte por doença cardíaca ${ }^{8}$.

Indivíduos com disfunção sexual frequentemente apresentam dúvidas e são influenciados pelos mitos populares, estabelecendo-se um ciclo vicioso que repercute na saúde e na qualidade de vida. Contudo, embora seja inquestionável a relação da satisfação sexual com a saúde e a qualidade de vida, as manifestações da sexualidade humana são desconsideradas por médicos e outros profissionais da saúde. Tal omissão é bastante influenciada pelo fato da temática, construída sócio-historicamente, estar permeada por tabus e preconceitos, como os mitos sobre o desempenho sexual, após eventos cardíacos.

$O$ retorno às atividades sexuais, após eventos cardíacos merece uma abordagem criteriosa, propiciando o retorno seguro dos pacientes à vida sexual e contribuindo para diminuir a elevada prevalência de disfunção sexual, entre pessoas acometidas por doenças cardiovasculares ${ }^{9,10}$. Assim, da mesma forma que recebem informações, visando ao retorno ao trabalho e ao engajamento em programas de exercícios, torna-se relevante que os pacientes recebam orientações pertinentes sobre atividade sexual ${ }^{11,12}$.

Por conta disto, esta breve revisão narrativa tem como objetivo compilar e discutir os principais mecanismos fisiológicos que contribuem para a saúde corpo e mente, e defender as as hipóteses fisiológicas que, potencialmente, tornam plausível considerar a dança de salão uma excelente opção quando se objetiva ao mesmo tempo o aprimoramento da função cardiovascular e sexual.

\section{Epidemiologia}

A prevalência de DE costuma ser superior a 60,9\% nos homens com doenças cardiopulmonares e metabólicas, sendo que, em cerca de 35,7\% ocorre disfunção sexual grave e igualmente preocupante na população feminina ${ }^{13,14}$. Aproximadamente, um terço das mulheres brasileiras apresentam desejo sexual hipoativo, 29\% não atingem o orgasmo e $18 \%$ têm dispaurenia ${ }^{15}$. Na população americana, a DS atinge $43 \%$ das mulheres e acomete $31 \%$ dos homens ${ }^{16}$. No Brasil, $45 \%$ da população, maior de 18 anos, apresenta algum grau de $\mathrm{DE}^{15,17}$, sendo $31,2 \%$ leve, 12,2 moderada e 1,7 severa $^{15}$ incidindo anualmente (novos casos) em um milhão de homens com idade entre 40 e 70 anos $^{17}$.

\section{Fatores de risco}

Os fatores de risco associados à disfunção sexual em homens e mulheres são comuns aos das doenças cardiovasculares, como, por exemplo, o sedentarismo, a obesidade, a hipertensão, a hipercolesterolemia, o diabetes e o tabagismo. Outros fatores, como as doenças do trato geniturinário, distúrbios psiquiátricos/psicológicos, doenças crônicas e condições sócio-demográficas, podem também prejudicar a função sexual.

A ocorrência de disfunção sexual pode diminuir por meio da modificação desses fatores de risco, merecendo destaque a prática de exercício físico e a perda de peso ${ }^{1}$. A incidência e a gravidade da disfunção erétil estão diretamente relacionadas à idade do paciente ${ }^{4}$.
A probabilidade de um indivíduo apresentar DE grave é aproximadamente três vezes maior aos 70 anos, quando comparada a indivíduos com 40 anos $(15 \%$ e $5,1 \%$ respectivamente) $)^{18}$.

Dentre os principais fatores de risco para DS, destaca-se a hipertensão arterial, sendo que esses indivíduos apresentam maior prevalência de disfunção sexual que os normotensos ${ }^{19}$, e a ocorrência e gravidade estão associadas ao grau da hipertensão, correlacionando-se positivamente com a pressão sistólica.

Nos pacientes com insuficiência cardíaca ocorrem complexas modificações hemodinâmicas, anatômicas, funcionais e biológicas que comprometem progressivamente a funcionalidade do sistema cardiovascular ${ }^{20-22}$ e, concomitantemente, a função sexual ${ }^{23}$.

$\mathrm{O}$ diabetes mellitus, a obesidade e o tabagismo, comprovadamente afetam a função sexual ${ }^{17,24,25}$. Deve-se levar em conta também a diminuição da aptidão cardiorrespiratória que dificulta a prática da atividade sexual, além de contribuir para a ansiedade relacionada ao desempenho e pode causar depressão. As doenças cardiovasculares, a disfunção sexual e a depressão são descritas como a tríade de condições que frequentemente agem em sinergia ${ }^{26}$.

0 tratamento farmacológico tradicional para as doenças cardiovasculares, por meio de diuréticos tiazídicos, digoxina, aldosterona e beta-bloqueadores, tem sido associado a $\mathrm{DE}^{23,27}$. No entanto, alguns fármacos de terceira geração, como beta-bloqueadores e bloqueadores dos receptores da angiotensina têm propiciado melhora da função sexual em mulheres e homens, devido seus efeitos de aprimoramento da circulação na esfera genital, em especial devido à melhora da função endotelial, por meio da maior liberação e menor degradação do oxido nítrico, de forma semelhante ao que ocorre com os fármacos facilitadores do sexo, usado em larga escala pela população masculina ${ }^{28,29}$.

\section{Tratamento}

Em homens, no tratamento convencional da disfunção erétil, é frequente o uso de inibidores da fosfodiesterase-5, que são os fármacos que aumentam a disponibilidade do oxido nítrico, a revascularização peniana, aconselhamento sexual e tratamento da depressão ${ }^{23}$. A terapia por meio da administração de hormônios também pode trazer benefícios para a função sexual. Nos homens o uso de testosterona está associado a pequenas melhorias na satisfação com a função erétil e melhora moderada na libido ${ }^{30}$. No sexo feminino os estrógenos promovem efeito sobre o trofismo vaginal, aliviam os quadros de dispareunia, secundários à atrofia do epitélio da vagina, visto que restauram esse epitélio, bem como o $\mathrm{pH}$ e o fluxo sanguíneo vaginais ${ }^{31}$. O seu efeito sobre o desejo é, pois, indireto. Entretanto, quando a falta de desejo não é secundária à dor ou ao desconforto durante o ato, o desinteresse sexual persiste, apesar da terapêutica estrogênica.

A prática de exercício físico, um potente ansiolítico e antidepressivo, merece atenção devido aos benefícios proporcionados na diminuição dos fatores de risco e melhora da capacidade cardiorrespiratória. O exercício também proporciona importante aumento da produção e diminuição da degradação de oxido nítrico, de forma semelhante ao proporcionado pelos fármacos 
facilitadores da ereção masculina, os inibidores da fosfodiesterase-5. Acrescenta-se ainda que o exercício físico contribui magnificamente para melhorar a autoimagem e a autoestima, proporcionado maior segurança à atividade sexual.

\section{Função sexual e exercício}

Vale lembrar que a resposta sexual em ambos os sexos é mediada por uma complexa interação entre fatores psicológicos, ambientais e fisiológico: (hormonais, vasculares, musculares e neurológicos). Acredita-se que os principais mediadores da função sexual masculina (óxido nítrico e catecolameninas) exercem os mesmos efeitos sobre o tecido genital feminino. A função erétil e dependente da liberação do óxido nítrico (NO) para promover vasodilatação e consequentemente permitir a ereção ${ }^{5,19}$. Na DE, a vasodilatação, óxido nítrico-dependente, costuma ficar gravemente prejudicada, em decorrência do desequilíbrio entre vasoconstrição e vasodilatação ${ }^{18}$.

Nesse contexto complexo, o exercício físico regular, com seus múltiplos efeitos, destaca-se como estratégia a ser considerada no tratamento da disfunção sexual, pois altera positivamente o funcionamento oxidativo das células e tecidos, aumentando os níveis de óxido nítrico que, por sua vez, aciona proteínas com função de reparo e prevenção de danos teciduais colaborando assim para melhora da função sexual, além dos seus efeitos psicológicos e sociais (maior autoestima, maior confiança no esforço físico, redução da ansieda-

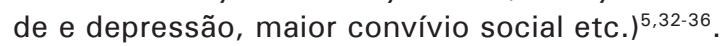

Dentre as modalidades de exercício físico, destaca-se a dança, que proporciona diversão, facilita a interação afetiva entre os parceiros ${ }^{37}$ favorece uma interação social positiva ${ }^{38}$, tendo sido evidenciados benefícios na função endotelial, aptidão cardiorrespiratória ${ }^{39,40}$, consumo pico de oxigênio(VO2pico) ${ }^{40,41}$, equivalente ven- tilatório de gás carbônico (VE/VCO2 slope) ${ }^{42}$, pressão arterial sistólica e diastólica ${ }^{41,43}$ que repercutem na melhora na qualidade de vida ${ }^{40,41,42}$. Acredita-se que indivíduos que praticam dança geralmente são mais motivados a manter aderência à atividade física do que participantes de programas de exercício convencional $^{40,41,42}$. No Núcleo de Cardiologia e Medicina do Exercício (NCME) da Universidade do Estado de Santa Catarina - UDESC vem desenvolvendo, nos últimos anos, no Programa de Reabilitação Cardiopulmonar e Metabólica, pesquisas com intuito de investigar os benefícios da dança de salão na saúde cardiovascular e função sexual de participantes do programa. Ao mesmo tempo, introduziu como forma alternativa de exercício, aulas de dança de salão, como meio de condicionamento físico, com adoção de ritmos variados (forró, bolero, samba, merengue, valsa, rock and roll e salsa), estas ministradas por professores especializados, para portadores de doenças cardiovasculares ${ }^{41}$ algo que está em sintonia com o que tem sido preconizado por sociedades médicas ${ }^{45}$. Mais que o ensino técnico dos passos, que exigiria frequentes interrupções, visamos manter os pacientes o maior tempo possível ativos, no intuito de sustentar a zona alvo da frequência cardíaca durante o exercício. Por meio dessa sistemática, temos sustentado resultados de maior aderência, com melhor resposta cronotrópica e controle da pressão arterial, fatores amplamente associados com disfunção sexual e desfechos cardiovasculares ${ }^{44}$.

Na tabela 1, é possível verificar pesquisas recentes desenvolvidas pelo grupo.

Nota-se que, em estudo inicial desenvolvido pelo grupo no ano de 2011, com pacientes hipertensos, o comportamento agudo da pressão arterial foi semelhante no dia sem exercício, após única sessão de caminhada e de dança de salão, não apresentando diferença significativa ${ }^{43}$.

Tabela 1 - Pesquisas do Núcleo de Cardiologia e Medicina do Exercício (NCME) envolvendo a utilização da dança de salão.

\begin{tabular}{|c|c|c|c|c|}
\hline AUTOR & OBJETIVO & Amostra & PROTOCOLO/DESIGN & Desfechos \\
\hline $\begin{array}{c}\text { Braga et al } \\
(2014) 46\end{array}$ & $\begin{array}{l}\text { Avaliar um protocolo de sam- } \\
\text { ba a ser adotado no treina- } \\
\text { mento físico de cardiopatas } \\
\text { participantes de programa de } \\
\text { reabilitação cardíaca. }\end{array}$ & $\mathrm{N}=15$ & 3 aulas de samba brasileiro & $\begin{array}{l}\text { Os pacientes se mantiveram acima de } 76 \% \text { do } \\
\text { tempo total na zona-alvo considerada ideal para } \\
\text { treinamento, com a FC situada entre o } 1^{\circ} \text { e o } 2^{\circ} \\
\text { limiar ventilatório, e percepção de esforço de leve } \\
\text { a moderada. }\end{array}$ \\
\hline
\end{tabular}

\begin{tabular}{llll}
\hline Gonzáles et & Avaliar a função sexual e a & $\mathrm{N}=102$ & Estudo transversal. Aplicação de \\
al (2014)47 & aptidão cardiorrespiratória de & questionários e TCP. \\
& coronariopatas e hipertensos & 3 Grupos: DS, RCPM e \\
& participantes RCPM e DS & sedentários
\end{tabular}

Homens e mulheres praticantes de DS demonstraram menor possibilidade de apresentar disfunção sexual $(O R=0.352 ; p=0.020 ; O R=$ $1.05 ; p=0.041$, respectivamente). O grupo DS apresentou VO2pico e VO2 no primeiro limiar ventilatório maior que os outros grupos (<0.001), e VO2pico $16 \%$ maior que o grupo RCPM e $21 \%$ maior que o GS.

\begin{tabular}{|c|c|c|c|}
\hline $\begin{array}{l}\text { Schenkel et } \\
\text { al, (2011)43 }\end{array}$ & $\begin{array}{l}\text { Avaliar o comportamento da } \\
\text { PA após uma sessão de DS e } \\
\text { de caminhada em indivíduos } \\
\text { hipertensos. }\end{array}$ & $N=8$ & $\begin{array}{l}3 \text { momentos diferentes: a) num } \\
\text { dia sem exercícios; b) após } 30 \\
\text { minutos de caminhada; e c) após } \\
\text { sessão de DS }\end{array}$ \\
\hline
\end{tabular}

Os valores médios de PAS e PAD no período total ( 22 horas), vigília e sono nas três diferentes situações não apresentaram diferença estatística. As curvas de PAS e PAD no decorrer das 22 horas pós-exercício de ambas as modalidades apresentaram melhor comportamento comparado ao dia sem exercícios, sendo mais evidente nas horas posteriores à sessão de DS, porém sem diferença estatística.

Legenda: RCPM - reabilitação cardiopulmonar e metabólica; DS - dança de salão; PA - pressão arterial; TCP - teste cardiopulmonar; FC - frequência cardíaca; VO2pico - consumo pico de oxigênio;VO2 - consumo máximo de oxigênio; GS - grupo sedentário; PAS: pressão arterial sistólica; PAD: pressão arterial diastólica . 
Em continuidade ao estudo dos efeitos da dança de salão em indivíduos com doenças cardiovasculares e metabólicos, estudo desenvolvido por Braga et al (2014) verificou que pacientes com doença arterial coronariana (DAC) se mantiveram acima de $76 \%$ do tempo total das aulas de samba na zona-alvo considerada ideal para treinamento, com a FC situada entre o $1^{\circ}$ e o $2^{\circ}$ limiar ventilatório, e percepção de esforço de leve a moderada. Neste estudo pode-se concluir que estes indivíduos demonstraram boa adaptação ao protocolo de Samba brasileiro, que se mostrou efetivo como proposta de treinamento na reabilitação cardíaca. Adicionalmente, estudo realizado no mesmo ano por Gonzáles et al (2014) verificou que homens e mulheres praticantes de dança de salão demonstraram menor razão de chance em desenvolver disfunção erétil e sexual, respectivamente.Adicionalmente, estes também apresentaram maiores valores para as variáveis $\mathrm{VO} 2$ pico e consumo de oxigênio (VO2) no primeiro limiar ventilatório (LV1), em comparação a indivíduos praticantes de reabilitação cardíaca convencional e indivíduos sedentários. Com estes dados, o estudo pode concluir que homens e mulheres, coronariopatas e hipertensos, praticantes de dança de salão possuem maior capacidade cardiorrespiratória e menor chance de disfunção sexual em comparação a participantes no programa de reabilitação convencional e sedentário.

\section{CONSIDERAÇÕES FINAIS}

São consistentes as informações científicas que ressaltam o papel do exercício físico na promoção da saúde e da qualidade de vida, contexto em que a saúde sexual tem relevância. Existe relação entre disfunção sexual e doenças cardiovasculares, sendo o exercício físico eficaz no tratamento de ambos. Estudos recentes vêm demonstrado que a dança de salão tem proporcionado efeitos cardiovasculares semelhantes aos exercício convencionais, com maior aprimoramento da capacidade cardiorrespiratória, maior motivação e aderência e menor chance de disfunção sexual. Neste sentido, torna-se plausível a hipótese de que a dança de salão pode ser considerada como excelente opção, quando se objetiva ao mesmo tempo o aprimoramento da função cardiovascular e sexual.

\section{REFERÊNCIAS}

1. Hatzimouratidis K, Amar E, Eardley I, Giuliano F, Hatzichristou D, Montorsi F et al. Guidelines on Male Sexual Dysfunction: Erectile Dysfunction and Premature Ejaculation. Eur Urol, 2010; 57(5): 804-14.

2. Mulhall J, King R, Glina S, Hvidsten K. Importance of and satisfaction with sex mong men and women worldwide: results of the global better sex survey. J Sex Med, 2008; 5: 788-95.

3. Studd J. A comparison of 19th century and current attitudes to female 2 sexuality. Gynecol Endocrinol, 2007; 23(12): 673-81.

4. NIH Consensus Conference. NIH Consensus Development Panel on Impotence. JAMA, 1993; 270: 83-90.

5. Lue TF, Giuliano F, Montorsi F, Rosen RC, Andersson
$\mathrm{KE}$, Althof $\mathrm{S}$ et al. Summary of the recommendations on sexual dysfunctions in men. J Sex Med, 2004; 1(1): 6-23.

6. Bachman GA, Dilek AVCl. Evaluation and management of female sexual dysfunction. Endocrinol, 2004; 14(6): 337-45.

7. Bernhard LA. Sexuality and sexual health care for women. Clin Obstet Gynecol, 2002; 45(4): 1089-98.

8. Schwartz BG, Kloner RA. Clinical Cardiology. Physician Update: Erectile Dysfunction and Cardiovascular Disease. Circulation, 2011; 123: 98-101.

9. Jackson G, Betteridge J, Dean J, Hall R, Holdright D, Holmes $\mathrm{S}$, et al. A systematic approach to erectile dysfunction in the cardiovascular patient: a consensus statement. Int $\mathrm{J}$ Clin Pract, 1999; 56(9): 445-51.

10. Symonds T, Boolell M, Quirk F. Development of a questionnaire on sexual quality of life in women. J Sex Marital Ther, 2005; 31: 385-97.

11. Rerkpattanapipat $P$, Stanek MS, Kotler MN. Sex and the heart: what is the role of the cardiologist? Eur Heart J, 2001; 22: 201-08.

12. Stein RA. Cardiovascular response to sexual activity. Am J Cardiol. 86(2A), 27F-29F, 2000.

13. Sties SW, Wittkopf PG, Panigas TF, Gonzáles Al, Cardoso FL, Carvalho T. Qualidade de vida e função sexual em participantes de programa de reabilitação cardiopulmonar e metabólica. Revista da Sociedadede Cardiologia do Estado de São Paulo, 22, 1-5, 2012.

14. Böhm M, Baumhäkel M, Probstfield JL, Schmieder R, Yusuf $\mathrm{S}$, Zhao $\mathrm{F}$, et al. Behalf of the ONTARGET/TRANSCEND EDInvestigators Seattle, WA; Homburg and Erlangen, Germany; and Hamilton, Ontario, Canada. Sexual function, satisfaction, and association of erectile dysfunction with cardiovascular disease and risk factors in cardiovascular high-risk patients: Substudy of the ONgoing Telmisartan Alone and in Combination with Ramipril Global Endpoint Trial/Telmisartan Randomized AssessmeNT Study in ACE-Intolerant Subjects with Cardiovascular Disease (ONTARGET/TRANSCEND) Am Heart J, 154, 2007.

15. Abdo CHN, Oliveira WM Jr, Scanavino MT, Martins FG. Disfunção erétil: resultados do estudo da vida sexual do brasileiro. Revista da Associação Médica Brasileira, 2006; 52(6): 424-9.

16. Laumann EO, Paik A, Rosen RC. The epidemiology of erectile dysfunction: results from the National Health and Social Life Survey. Int J Imp Res. 11,Suppl 1,S60-4, 1999.

17. Moreira ED, Abdo CHN, Wroclawski E, Fittipaldi JAS. Epidemiologia da disfunção erétil no Brasil: resultados da pesquisa nacional do "Projeto Avaliar". Revista Brasileira de Medicina, 2004; 9: 613-25.

18. Feldman HA, Goldstein I, Hatzichristou DG, Krane RJ, McKinlay JB. Impotence and its medical and psychosocial correlates: results of the Massachussets Male Aging Study. J Urol, 151, 54-61, 1994.

19. Manolis A, Doumas M. Sexual dysfunction: the 'prima ballerina' of hypertension related quality-of-life complications. J Hyperten, 2008; 26(11): 2074-84.

20. Seixas-Cambão M, Leite-Moreira A. Fisiopatologia da Insuficiência Cardíaca Crónica. Revista Portuguesa de Cardiologia, 2009; 28: 439-71.

21. Serna F. Aspectos generales de la fisiopatología de la insuficiencia cardiaca. In: Serna, F. Insuficiencia cardíaca crônica. Editorial Federación Argentina de Cardiología, Buenos Aires,n.3, 2010. Disponível em: <http://www.fac.org.ar/ edicion/inscac/cap02 2010.pdf. Acesso: 02 dez.2011. 
22. Balakumar $P$, Jagadeesh $G$. Multifarious molecular signaling cascades of cardiac hypertrophy: can the muddy waters be cleared? Pharmacol Res, 2010; 62(5): 365-83.

23. Schwarzer, Rastogi S, Kapur V, Suelemanjee N, Rodriguez JJ. Erectile dysfunction in heart failure patients. J Am Coll Cardiol. 48, 1111-19, sept. 2006.

24. Braun M, Wassmer G, Klotz T, Reifenrath B, Mathers $M$, Engelmann U. Epidemiology of erectile dysfunction: results of the 'Cologne Male Survey'. Int J Impot Res, 2000; 12: 305- 11

25. Bornman MS, Du Plessis DJ. Smoking and vascular impotence. A reason for concern. South African Medical Journal, 1986; 70(6): 329-30.

26. Goldstein I. The mutually reinforcing triad of depressive symptoms, cardiovascular disease, and erectile dysfunction. Am J Cardiol. 86, 41F-45F, 2000.

27. Muguruma $H$, Kawanishi $Y$, Sugiyama $H$, Kagawa J, Tanimoto S, Yamanaka M, et al. Effect of aldosterone on isolated human penile corpus cavernosum tissue. BJU Int, 2008;102(4): 500-503.

28. Brixius K, Middeke M, Lichtenthal A, Jahn E, Schwinger RH. Nitric oxide, erectile dysfunction and beta-blocker treatment (MR NOED study): benefit of nebivolol versus metoprolol in hypertensive men. Clin Exp Pharmacol Physiol, 2007; 34: 327-31.

29. Toblli JE, Cao G, Casas G, Mazza ON. In vivo and in vitro effects of nebivolol on penile structures in hypertensive rats. Am J Hyperten. 2006; 19: 1226-32.

30. Boloña ER, Uraga MV, Haddad RM, Tracz MJ, Sideras K, Kennedy CC, et al. Testosterone Use in Men With Sexual Dysfunction: A Systematic Review and Meta-analysis of Randomized Placebo-Controlled Trials. Mayo Clin Proc, 2007; 82(1): 20-28.

31. Fernandes CE. A influência dos hormônios na sexualidade feminina no climatério pós-menopáusico. Revista Médico Reporter, 2005; 7(65):14-18.

32. Derby CA, Mohr BA, Goldstein I, Feldman HA, Johannes $\mathrm{CB}$, McKinlay JB. Modifiabble risk factors and erectile dysfunction: can lifestyle changes modify risk? Urology, 2000; 56(2): 302-6.

33. Bacon CG, Mittleman MA, Kawachi I, Giovannucci E, Glasser DB, Rimm EB. Sexual function in men older than 50 years of age: results from the health professionals follow-up study. Ann Intern Med, 2003; 139(3):161-8.

34. Agostini LC, Netto JM, Miranda MV Jr, Figueiredo AA. Erectile dysfunction association with physical activity level and physical fitness in men aged 40-75 years. Int $\mathrm{J}$ Impot Res, 2011; 23(3): 115-21.
35. Working Group on Cardiac Rehabilitation \& Exercice Physiology and Working Group on Heart Failure of the European Society of Cardiology. Recommendations for exercise training in chronic heart failure patients. Eur Heart $\mathrm{J}$, 2001; 22(2): 125-35.

36. Lloyd-Williams F, Mair FS, Leitner M. Exercise training and heart failure: a systematic review of current evidence. $\mathrm{Br} J$ Gen Pract, 2002; 52(474): 47-55.

37. Reid B. Fundamentos da dança de salão: Programa Internacional de Dança de Salão. Londrina: Midiograf, 2003.

38. Haboush A, Floyd M, Caron J, Lasota M, Alvarez K. Ballroom dance lessons for geriatric depression: an exploratory study. The Arts in Psychotherapy, 2005; 33(2): 1-9.

39. Costa J, Dias C, Gonçalves D, Pereira MM, Safons MP, Baldissera V. Duplo produto como variável de segurança para a prática de dança de salão em idosos. Revista Digital, 13(120), 2008.

40. Belardinelli R, Lacalaprice F, Ventrella C, Volpe L, Faccenda E. Waltz dancing in patients with chronic heart failure: new form of exercise training. Circ Heart Fail. 1, 107-14, 2008.

41. Aweto HA, Owoeye OB, Akinbo SR, Onabajo AA. Effects of dance movement therapy on selected cardiovascular parameters and estimated maximum exygen consumption in hypertensive patients. Nig Q J Hosp Me.2012;22(2)125-9. (Abstract).

42. Kaltsatou ACH, Kouidi El, Anifanti MA, Douka SI, Deligiannis AP.

43. Functional and psychosocial effects of either a traditional dancing or a formal exercising training program in patients with chronic heart failure: a comparative randomized controlled study. Clin Rehabil. 2013;28(2):128-38.

44. Schenkel IC, Bündchen DC, Quites MP, Santos RZS, Santos MB, Carvalho T. Comportamento da pressão arterial em hipertensos após única sessão de caminhada e de dança de salão: estudo preliminar. Revista Brasileira de Cardiologia, 2011; 24(1): 26-32.

45. Carvalho T, Gonzáles Al, Sties SW, Carvalho MGD. Reabilitação Cardiovascular, dança de salão e disfunção sexual. Arq Bras Cardio, 2013; 101(6): 107-108.

46. Carvalho T. Posição oficial da Sociedade Brasileira de Medicina do Esporte: atividade física e saúde. Revista Brasileira de Medicina do Esporte, 1996; 2(4): 79-81.

47. Braga HO, Gonzáles Al, Sties SW, Carvalho GD, Neto AS, Campos OA, et al. Protocolo de Samba Brasileiro para reabilitação cardíaca. 2014 (artigo submetido para publicação)

48. Gonzáles Al, Braga HO, Sties SW, Mara LS, Carvalho GMD, Souza AV et al. Função sexual e aptidão cardiorrespiratória de coronariopatas e hipertensos praticantes de dança. 2014 (artigo submetido para publicação). 